\title{
Erratum: 'All-optical switching in the directional coupler caused by nonlinear refraction due to cascaded second-order nonlinearity'
}

R. SCHIEK, Optical and Quantum Electronics 26 (1994) 415.

All power density units $\mathrm{MWm}^{-1}$ are erroneously printed as $\mathrm{mWm}^{-1}$. Wherever $\mathrm{mWm}^{-1}$ appears it should read $\mathrm{MWm}^{-1}$. 